\title{
Reliable and Secure Surveillance, Communications and Navigation (RSCAN) for Unmanned Air Systems (UAS) in Controlled Airspace
}

\author{
Denise S. Ponchak \\ NASA Glenn Research Center \\ 21000 Brookpark Rd. MS 54-1 \\ Cleveland, OH 44135 \\ denise.s.ponchak@nasa.gov
}

\author{
Fred L. Templin \\ The Boeing Company \\ P.O. Box 3707 MC 42-59 \\ Seattle, WA 98124 \\ Phone: 425-373-2829 \\ fred.l.templin@boeing.com
}

\author{
Greg Sheffield \\ The Boeing Company \\ P.O. Box 516 MC S064-2374 \\ St Louis, MO 63166-0516 \\ Phone: 314-302-7543 \\ greg.l.sheffield@boeing.com
}

\author{
Pedro Taboso \\ The Boeing Company \\ Avenida Sur del Aeropuerto de \\ Barajas, 38 \\ 28042 - Madrid; Spain \\ Phone: +34 917688451
}

pedro.tabosoballesteros@boeing.com

\author{
Raj Jain \\ Washington University in St. Louis \\ Saint Louis, MO 63130 \\ Phone: +1 314-322-8092 \\ jain@acm.org
}

\begin{abstract}
The aviation industry faces a rapidly-emerging need for integrating Unmanned Air Systems (UAS) into the national airspace (NAS). This trend will present challenging questions for the safe operation of UAS in controlled and uncontrolled airspaces based on new Communications, Navigation and Surveillance (CNS) technologies. For example, can wireless communications data links provide the necessary capacity for accommodating ever increasing numbers of UAS worldwide? Does the communications network provide ample Internet Protocol (IP) address space to allow Air Traffic Control (ATC) to securely address each UAS? Can navigation and surveillance approaches assure safe route planning and safe separation of vehicles even in crowded skies?
\end{abstract}

Under NASA contract NNA16BD84C, Boeing is developing an integrated CNS architecture to enable UAS operations in the NAS. Revolutionary and advanced CNS alternatives are needed to support UAS operations at all altitudes and in all airspaces, including both controlled and uncontrolled. These CNS alternatives must be reliable, redundant, always available, cyber-secure, and affordable for all types of vehicles including small UAS to large transport category aircraft. Our approach considers CNS requirements that address the range of UAS missions where they will be most beneficial and costeffective.

A cybersecure future UAS CNS architecture is needed to support the NASA vision for an Unmanned Air Traffic Management (UTM) system in uncontrolled airspace and a cooperative operation of manned and unmanned aircraft in the controlled global Air Traffic Management (ATM) system. The architecture must, therefore, support always-available and cyber secure operations. This paper presents UAS CNS architecture concepts for large UAS operating in the ATM system in controlled airspace. Future companion works will consider small UAS operating in the UTM system in uncontrolled airspace.

\section{TABLE OF CONTENTS}

1. INTRODUCTION 1

2. COMMUNICATIONS - NETWORKS ............................2

3. COMMUNICATIONS - DATA LINKS ........................5

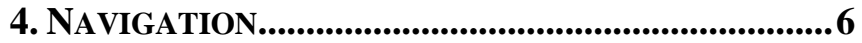

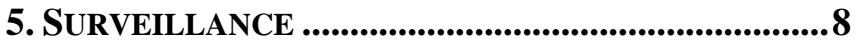

6. SUMMARY ........................................................11

ACKNOWLEDGEMENTS .............................................11

REFERENCES .....................................................11

\section{INTRODUCTION}

This study considers new architectural concepts for the safe operation of Unmanned Air Systems (UAS) in controlled airspace (i.e., airspace categories $\mathrm{A} / \mathrm{B} / \mathrm{C} / \mathrm{D} / \mathrm{E}$ ). The significant implication of this prospect is that UAS will need to share the same airspace as for manned aviation. This will require revolutionary new architectural approaches to Communications, Navigation and Surveillance (CNS) to ensure that UAS can be integrated safely into worldwide Air Traffic Management (ATM) system.

The tremendous growth of UAS in air traffic is anticipated to put a strain on airspace capacity and airport resources. To mitigate the growth, a new architecture will be required to take advantage of emerging CNS technologies. To implement the improvements, the air traffic system requires significant upgrades to increase system capacity and flight efficiency while continuing to meet flight safety standards.

The air traffic CNS architecture supporting the growing number of UAS platforms in the NAS will require new integrated solutions onboard and additional enterprise CNS systems functionality. 
Figure 1 shows a notional CNS architecture to support the integration of new technologies onboard each UAS platform and the integration of advancements in existing enterprise systems. while the ground pilot issues Control and Non-Payload Communication (CNPC) directives to the UA. For current ATM systems in manned aviation, primary communications are through analog voice with data link short text messaging as a secondary facility. Therefore, ATC/AOC must be able

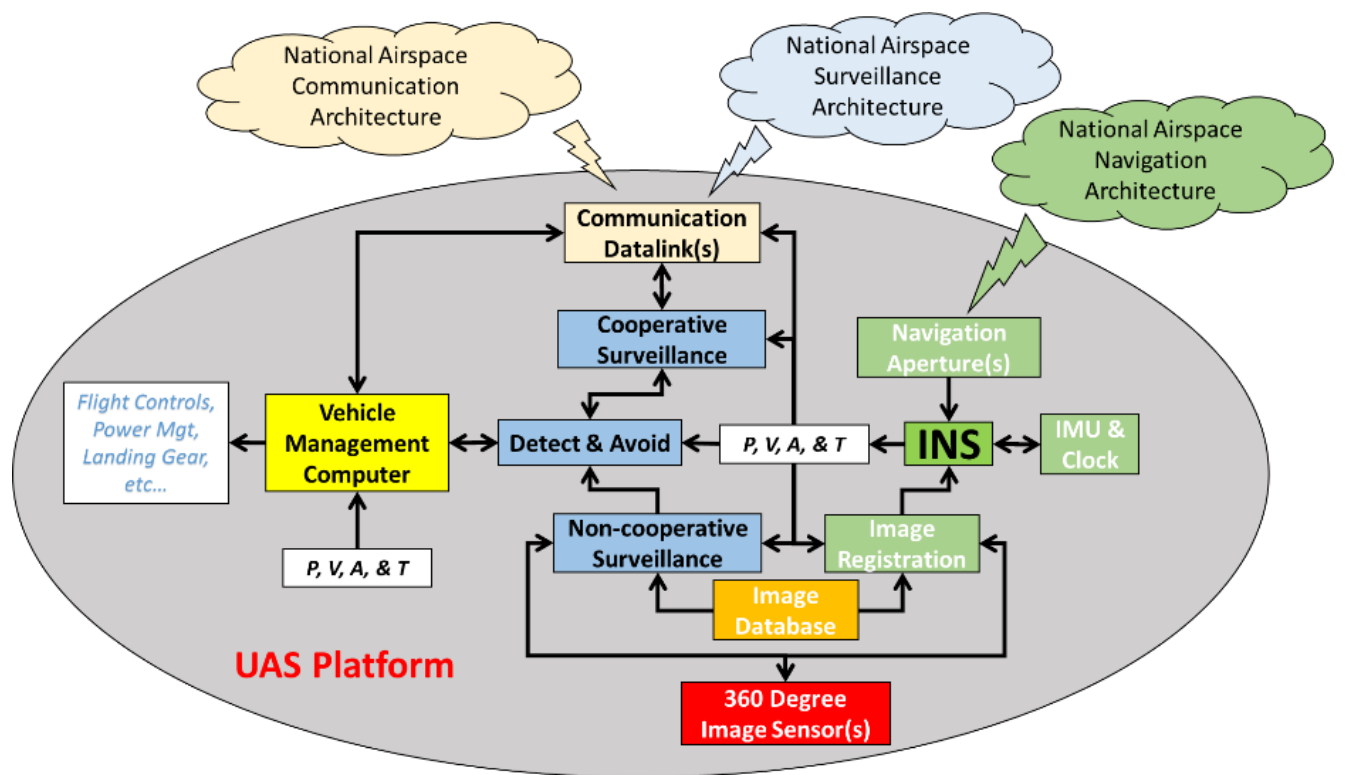

Figure 1 - Combined UAS CNS Architectural Framework

Navigation augmentations and surveillance information will be integrated with the communications network and data link services so that UAS can operate safely in controlled airspace in cooperation with manned aviation in the ATM system. Data communications will further provide the critical infrastructure for command and control (C2), situation awareness (SA), navigation and surveillance. Therefore, each element is an interdependent component of the integrated CNS architecture much in the same way that the engine, transmission, chassis, body and wheels are all interdependent components of an automobile. In the following sections, we discuss the constituent elements of the proposed integrated UAS CNS architecture in further detail.

\section{COMMUNICATIONS - NeTWORKS}

\section{Introduction}

Unmanned Aircraft (UAs) operating in controlled airspace will come under the same ATM jurisdiction as for manned aviation. These UAs will occupy Class A/B/C/D/E airspace such that their operations will be in non-segregated airspace where manned aircraft also operate. This means that the UAS will require a robust and highly-available networked communications system for Air Traffic Controllers (ATC) and Airline Operations Controllers (AOC) to issue ATM directives at any time and with high reliability.

Where manned aviation differs from UAS operations is that the pilot is on the ground and not on board the aircraft. This means that there will be a communications profile triad in which the ATC/AOC issues directives to the ground pilot, to communicate with the ground pilot the same as for manned aviation, and the ground pilot must act on the ATC/AOC directives by appropriately directing the UA.

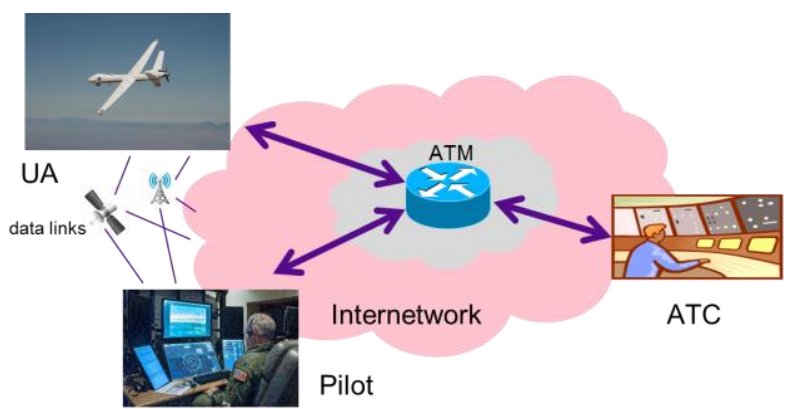

Figure 2 - UA/Pilot/ATC Communications Triad

This UAS communications paradigm (Figure 2) has parallels to the way current-day Department of Defense (DoD) operations of UAS are coordinated. Ground pilots in the continental United States control UAs operating in overseas theaters of operation. The ground pilot's CNPC workstation has a direct uplink connection to the satellite system which then has a direct downlink connection to the UA, with perhaps one or more relay satellites in the path. Furthermore, there must be a separate secured voice and/or data coordination channel for mission commanders to direct the pilot.

The ground pilot could be located at any physically-secured location worldwide from which secured UA data link connections and secured ATM coordination channels are available. It is also possible to conceive of scenarios where a 
single UA is handed off between multiple ground pilots during the course of the flight.

While current-day ATM communications still rely on voice as a primary for both manned and unmanned aviation, the International Civil Aviation Organization (ICAO) is actively working toward a data communications system known as the Aeronautical Telecommunications Network (ATN) with Internet Protocol Services (ATN/IPS) [1]. This system is planned to support data communications as the primary service, with voice as a backup service beginning in the 2025 and beyond timeframe. In the ICAO vision, a worldwide ATM internetworking service based on Internet
The ATN/IPS itself will be organized as a global enterprise network overlay for ATM services in the same manner that major multi-national corporations operate global enterprise networks to support their businesses. It will, therefore, require a strongly secured perimeter through physical, link, network and/or transport layer securing technologies. Security perimeter infrastructure such as Virtual Private Network (VPN) gateways and Subnetwork Border Routers (SBRs) connect Data link Service Provider (DSP) subnetworks to the ATN/IPS; each of which may provide connectivity to large numbers of UAs and other ATN/IPS end systems.

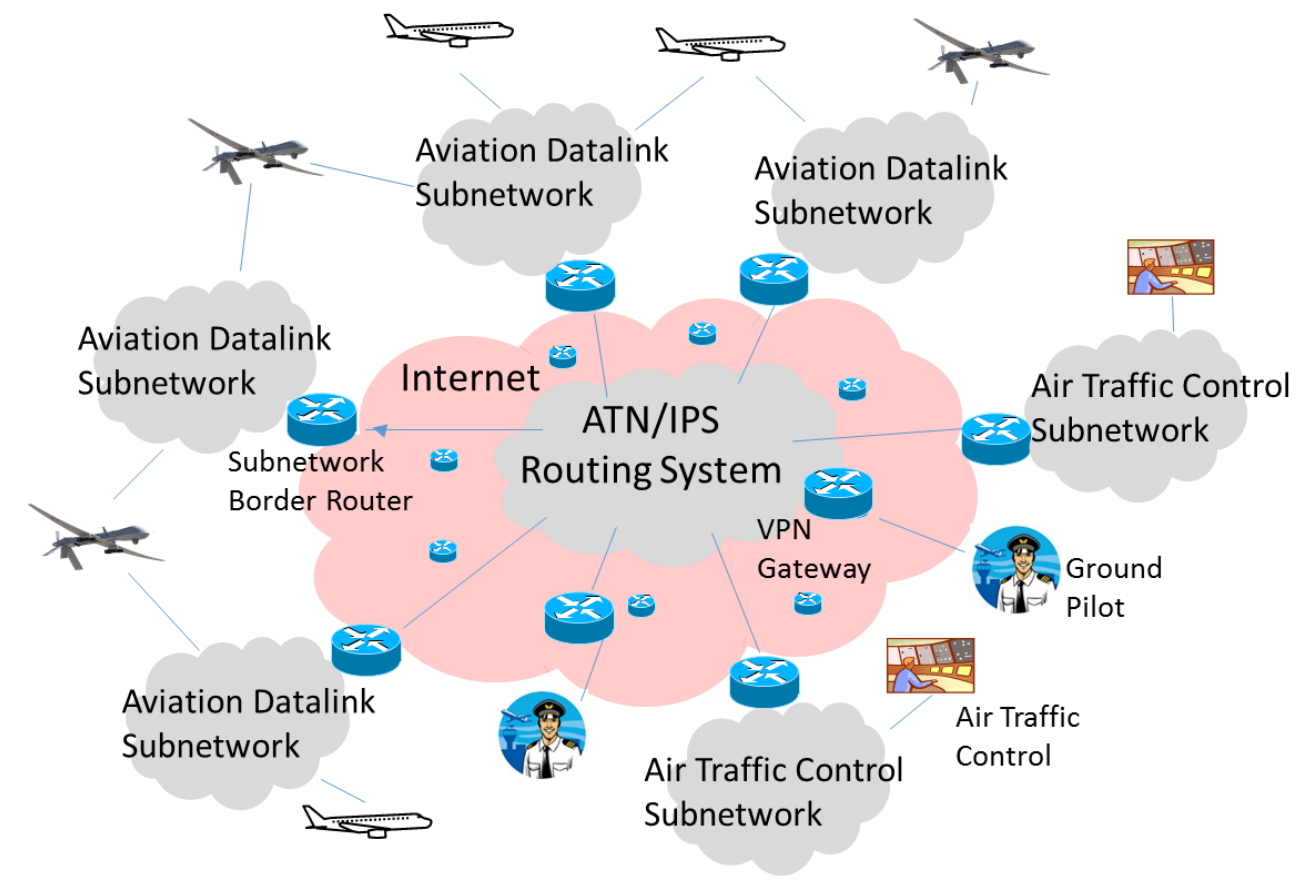

Figure 3 - ATN/IPS Internetwork

Protocol, version 6 (IPv6) messaging will be made available. This system is one and the same as that anticipated for future operations of UAS in controlled airspace and is the subject for the rest of this section.

\section{ATN/IPS Overview}

The ATN/IPS internetwork (Figure 3) will be configured as an overlay service (shown in gray) layered on top of the global public Internet (shown in pink) and/or interconnected by dedicated ground domain communications links such as high-speed fiber-optics. It will further be complementary to the Unmanned Air Traffic Management (UTM) system currently under consideration for small UAS operating in uncontrolled airspace [2]. The ATN/IPS will be constructed using Wide Area Networking (WAN) technologies such as Multi-Protocol Label Switching (MPLS-WAN) and Software-Defined WAN (SD-WAN). Any paths that utilize the underlying Internet as transit must be secured with Internet Protocol Security (IPsec) or similar network encryption technologies.
Internally, the ATN/IPS will allow global IPv6 addressing within an ATN/IPS Service Prefix (ASP) (for example, 2001:db8::/32) that is assigned by an Internet assigned numbers authority for the exclusive use of the ATN/IPS. Each ASP contains many Mobile Network Prefixes (MNPs) that are delegated to ATN/IPS clients and only made reachable among correspondents that are securely attached to the ATN/IPS - open Internet communications to MNP addresses must be heavily filtered and/or blocked by firewalls. In a fully segregated arrangement, there may be no open Internet connections allowed to or from the ATN/IPS at all.

ATN/IPS clients such as UAs act as mobile networks that can each be delegated one or more MNP. Each MNP travels with the client wherever it goes and provides a constant and unchanging IPv6 prefix that the client can use to number its internal devices, e.g., as a mobile Internet-of-Things (IoT).

UAs will normally connect to multiple aviation data links (e.g., satellite, terrestrial, cellular, etc.) at the same time. 
This multilink arrangement affords the best reliability, cost, performance and quality of service parameters throughout the UA's various phases of flight. In the ATN/IPS design, the multilink capability is also fundamentally tied to the concept of multihoming where the UA can be registered with multiple service provider networks at the same time. This arrangement provides greater reliability since the UA will have multiple data links to choose from

\section{ATN/IPS Internetworking Architecture}

Figure 4 shows the ATN/IPS multilink internetworking architecture. The service consists of the ATN/IPS physically connected underlay and with the Asymmetric Extended Route Optimization (AERO) [3] service as an overlay. The AERO overlay is responsible for mobility, multihoming, security, traffic engineering and quality-of-service based routing.

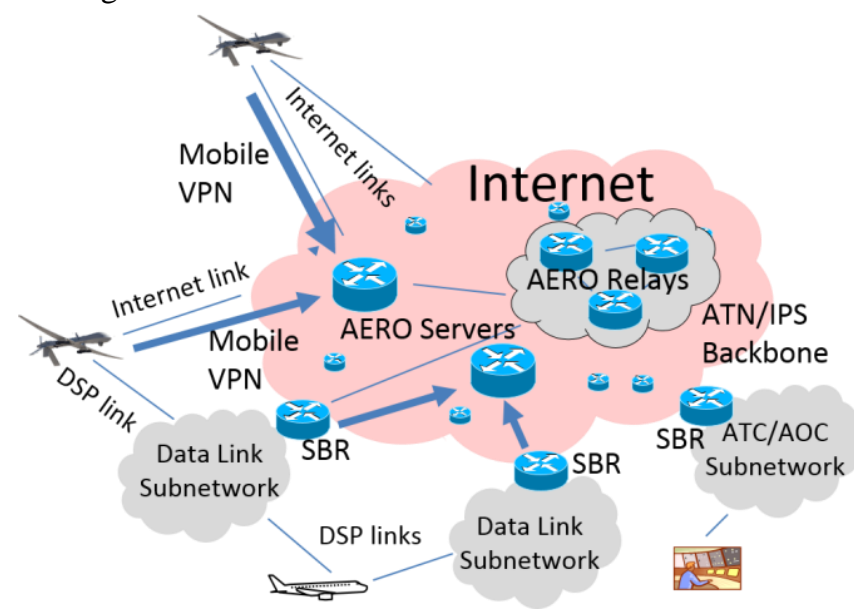

Figure 4 - ATN/IPS Internetworking Architecture

In this architecture, AERO Servers form the boundary of the ATN/IPS global enterprise network and all communications within the ATN/IPS are carried through encapsulation-based tunneling across the underlying internetwork. AERO Servers connect AERO Clients to the ATN/IPS (shown here as manned and unmanned aircraft) and can forward packets to destinations via AERO Relays (shown here in the ATN/IPS core).

Aircraft connect to the ATN/IPS via data links that may be terminated in a secured data link service provider network or open to the global public Internet. In the former case, data link service provider SBRs provide a proxy connectivity service to the Clients, while in the latter case the Clients maintain their own mobile VPNs.

From the interior viewpoint of the ATN/IPS global enterprise network, all AERO Servers present identical Client services, and Clients can associate with one or more Servers that are nearby. Each AERO Server provides Clientdirected Quality of Service (QoS) mappings and also provides priority and/or weight metrics so that AERO Relays can make forwarding decisions.
From an exterior viewpoint, each subnetwork model has its own manner of offering secured services to mobile clients, with each model having application for various ATN/IPS use cases. For example, large UAs operating over tightlymanaged DSP link types will likely use the closed subnetwork model. ATN/IPS correspondents on the open Internet will use the non-subnetwork model and connect directly to AERO Servers via a mobile VPN.

In the AERO model, AERO Servers and Relays participate in a private Border Gateway Protocol (BGP) instance that tracks all of the MNPs currently active in the ATN/IPS routing system. AERO Relays maintain a core Autonomous System (AS), while AERO Servers form stub ASes. When a source AERO Server has a packet to send, it sends the packet immediately via a default route to an AERO Relay which then forwards it toward the highest-priority target AERO Server. The source AERO Server can then initiate a route optimization procedure to discover one or more targets that it can send subsequent packets to without having to continue sending them through the dogleg path via the AERO Relays. This route optimization can be deferred until the direct path between the source and target AERO Servers can be tested so that the risk of black-holing along the path is eliminated.

In the AERO model, route optimization is through control message signaling after initial packets are successfully sent via the default route. An extremely important consideration, however, is whether a route optimized path can be considered usable before being tested. Instead of making a leap of faith, the source AERO Server tests the route optimized path first while data packets are allowed to continue to flow through a longer path that is known to work. The longer path can be considered more reliable since it travels over the same paths where BGP Transmission Control Protocol (TCP) session keepalives maintain reachability.

The route-optimized path between the source and target AERO Servers can fail at some time after the path was first tested. This means that some form of unreachability detection is required that can quickly detect the path failure with a minimum amount of packet loss. Furthermore, if an ATN/IPS end system moves from a first AERO Server to a second Server, any peers with a route optimization pointing to the first AERO Server will have to be informed that the route is no longer valid. If each AERO Server remembers the peers to which it previously sent route optimization messages, it can proactively send updates to invalidate the previous route optimizations. If the source AERO Server has not received an update and continues to send packets to the target, however, the target can reactively send "Destination Unreachable" messages while dropping the packets. From a reliability standpoint, therefore, it is very important that AERO Servers send proactive updates before any data packets arrive that would trigger a reactive Destination Unreachable. 


\section{Communications - Data Links}

\section{Current Data links for Controlled Airspace}

Currently, the aeronautical standard bodies are discussing two new data links for unmanned aircraft systems. These are Aeronautical Mobile Airport Communication System (AeroMACS) and L-band digital aeronautical communications system, type 1 (LDACS1). These are briefly described below along with a next-generation satellite system before discussing the ideas for the next generation.

AeroMACS - AeroMACS is the data link technology developed by RTCA SC-223 for airport surface communications. AeroMACS operates in the Aeronautical Mobile (R) Service (AM(R)S) allocation in the $C$ band. Specifically, a $55 \mathrm{MHz}$ band (5.095-5.150 GHz) is used and divided into eleven $5-\mathrm{MHz}$ channels. Each channel uses orthogonal frequency division modulation (OFDM) using 512 subcarriers with an inter-carrier spacing of approximately $10 \mathrm{kHz}$.

LDACS1 - LDACS1 is leading proposed alternatives for communications during in-flight phase (Mission category D2). It uses OFDM in the L band. Since L Band is lower in frequency than $\mathrm{C}$ band used in AeroMACS, it can reach farther distances. It uses 50 subcarriers in a $498 \mathrm{kHz}$ frequency band. The sub-carrier spacing of $9.76 \mathrm{kHz}$ in $\mathrm{L}-$ DACS1 is similar to that of WiMAX. For an in-depth analysis of L-DACS1, please see our papers [4][5][6].

Satellite Systems - Currently Inmarsat and Iridium-Next are cornerstones of the long-term plan for data communications for manned aircraft and we should expect the same links for UAS data comms in controlled airspace.

Another promising next-generation system is the SpaceX, which promises the lowest latency and highest data rate of all systems till to date. SpaceX is a collaboration of SpaceX corporation and Google. It plans to put 4000 Low-Earth Orbit (LEO) satellites by 2020. Using a $650 \mathrm{~km}$ orbit and inter-satellite links, it plans to offer a latency of $20 \mathrm{~ms}$. With 50 Gbps per satellite, the total throughput could over 200 Tbps. Assuming 50 million customers, the throughput per customer will be $4 \mathrm{Mbps}$. This should be ideal for UAS communication.

\section{Enhancements to ATM Data links}

The key metrics for UAS data links are peak data rate, per user data rates, and energy efficiency. Figure 5 shows the desired enhancements in these metrics.

Most forecasts of UAs in the controlled airspaces are based on those of the current manned aircraft and the increase in the number of total aircrafts even after ten years is forecasted to be less than $5 x$ [7]. This may be an underforecast since most of the applications of UAs are not in the same area as the manned aircrafts but in applications that currently use cars and trucks.
Given this uncertainty, we believe ideas that improve the data link performance by an order magnitude would be reasonable. Some of this increase will come from an increased spectrum that is being discussed in various international standards bodies. The remaining increase will have to come from increased spectral efficiency.

Similarly, assuming a 5-fold increase in UA density, per UA data rate will go up by a factor of 2 . This is in line with RTCA forecasts of data rate requirements for UA data links [8].

The next generation of ATM data links can benefit from some of the new Radio Multiplexing techniques that are being developed for the next generation of data communications.

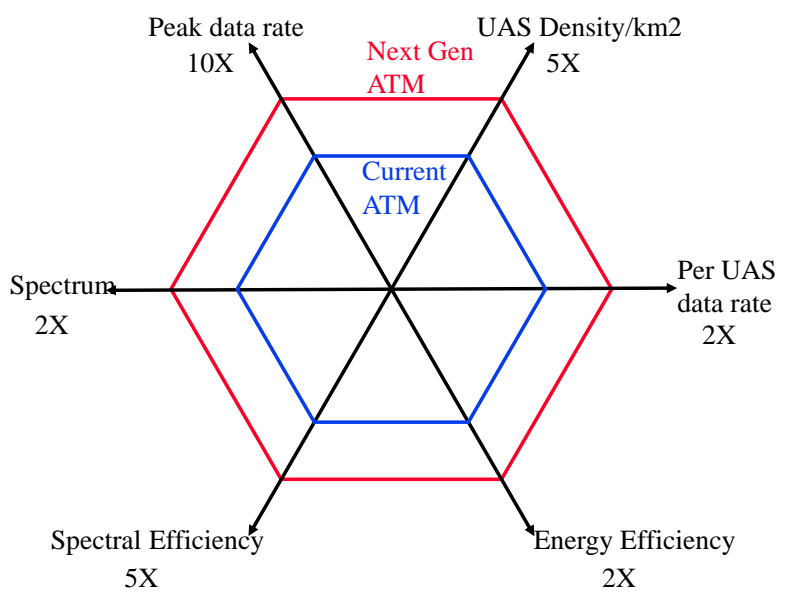

Figure 5 - Goals for Next Generation ATM Data Links

\section{New Radio Multiplexing Technologies}

Both LDACS-1 and AeroMACS use OFDM. Almost all wireless technologies developed in 2000-2010 use OFDM. OFDM is now known to have several problems [9] that newer proposals are aiming to solve. The problems with OFDM are:

1. Spectrum Overflow: In order to guarantee orthogonality, each subcarrier should have a zero power at the neighboring subcarriers. This results in a power ripple and there is a significant spectrum overflow beyond the spectrum used by the subcarriers. This is overcome in OFDM by having a guard band.

2. Same Subcarrier Spacing: OFDM requires that all subcarriers be equally spaced. To avoid a noisy frequency, an integral number of subcarriers need to be either not used or used at a low rate modulation.

3. Same Symbol Size: All subcarriers need to use the same symbol size and cyclic prefix. 
4. Time Synchronization: In OFDMA, all users should time synchronize in the uplink otherwise they will interfere with each other.

Newer technologies that overcome these problems are now being proposed. Two of these techniques are discussed in this section.

Spectrum Filtered OFDM (f-OFDM) - In f-OFDM, the frequency band is divided into multiple sub-bands and each sub-band may use different OFDM parameters (frequency spacing, cyclic prefix, symbol size) optimized for the applications. This is shown in Figure 6.

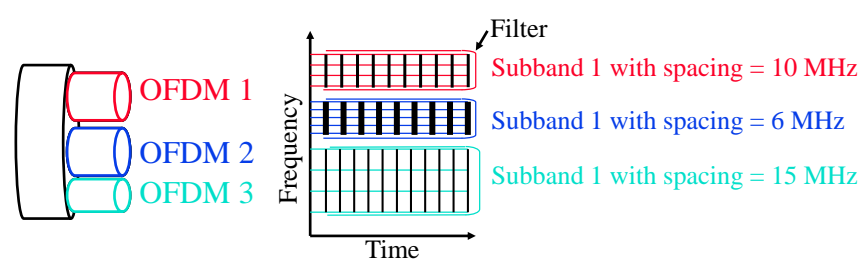

Figure 6 - Spectrum Filtered OFDM

For example, the next generation of AeroMACS (henceforth, called AeroMACS2) could use this technique to allow it to be used for ground applications and some part of taxi and takeoff where higher speed would require a different set of OFDM parameters. It could also be used to cover different areas of an airport differently. Aircrafts far away from the base station would use not only different rate modulation, but an entirely new set of OFDM parameters.

In f-OFDM, each sub-band is filtered to avoid inter-subband interference. Hence the name "Spectrum-filtered OFDM." Note that users of different sub-bands do not need to be time synchronized.

This technique can also be used with LDACS1 since it also uses OFDM. Again, different sub-bands will allow an optimized group of OFDM sub-bands for UAs at different distances from the base station or different speeds.

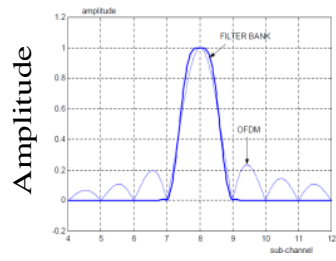

Frequency

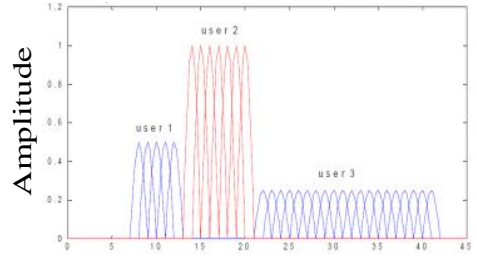

Frequency
Figure 7 - Filtered Bank Multicarrier (FBMC)

\section{Filtered Bank Multicarrier (FBMC)}

In this technique, a filter is used to remove the subcarrier overflow as shown by the thicker line in Figure 7. Since there are no side lobes, no cyclic prefix is needed and this allows more bits per Hertz. Again, this can be combined with multiple sub-bands as discussed above and different aircrafts can use different sub-bands with different OFDM parameters.

\section{NAVigation}

Regardless of Unmanned Aerial Systems (UAS) size and mission, all UASs share the need for navigation accuracy supporting guidance and control within a given airspace (e.g., Class A - G). Navigation accuracy serves as an input reference for non-cooperative surveillance by fusing multiple sensor sources to support detect and avoid capabilities. Plus, the navigation accuracy supports cooperative reporting of own-platform position, course, and speed.

Ground-controlled and autonomous operations of UASs require continuous and accurate measurements of the vehicle's position, velocity, attitude (orientation), and timing. Existing UAS's ground station controllers rely on GPS for determining position and velocity, plus determine attitude using a GPS aided Inertial Navigation Systems (INS) with the use of an IMU (Inertial Measurement Unit). This means that existing ground station controllers will have difficulty navigating, guiding, and controlling UASs when GPS is unavailable or degraded [10].

This section of the report is focused on navigation architectural concepts for UAS within controlled airspace. The discussion of navigation architecture concepts will factor in identified requirements which are listed in Table 1:

\section{Table 1 - Navigation Requirements}

\begin{tabular}{|l|l|}
\hline $\begin{array}{l}\text { Navigation } \\
\text { Requirements }\end{array}$ & Description \\
\hline $\begin{array}{l}\text { NV1: GPS } \\
\text { Augmentation }\end{array}$ & $\begin{array}{l}\text { To deal with GPS-denied } \\
\text { condition, an alternate navigation } \\
\text { system to augment GPS is } \\
\text { needed. }\end{array}$ \\
\hline $\begin{array}{l}\text { NV2: Certifiable } \\
\text { Computing } \\
\text { Architecture }\end{array}$ & $\begin{array}{l}\text { Cost affordable certifiable UAS } \\
\text { safety of flight computing } \\
\text { architecture to support navigation } \\
\text { algorithms on any size UAS } \\
\text { operating within NAS. }\end{array}$ \\
\hline $\begin{array}{l}\text { NV3: Navigation } \\
\text { Detection and } \\
\text { Correction }\end{array}$ & $\begin{array}{l}\text { Real-time error detection and } \\
\text { dynamic switching between } \\
\text { navigation sources to maintain } \\
\text { continuous position accuracy. }\end{array}$ \\
\hline $\begin{array}{l}\text { NV4: Ground } \\
\text { Controlled UAS } \\
\text { Navigation Accuracy } \\
\text { Schema }\end{array}$ & $\begin{array}{l}\text { UAS flight profiles and system } \\
\text { defined to properly control UAS } \\
\text { via ground station. }\end{array}$ \\
\hline $\begin{array}{l}\text { Nessage } \\
\text { Nessage types need to be defined } \\
\text { within STANAG 4586 to support } \\
\text { the exchange of navigation } \\
\text { information within NAS. }\end{array}$ \\
\hline
\end{tabular}




\begin{tabular}{|l|l|}
\hline $\begin{array}{l}\text { NV6: BLOS } \\
\text { Navigation Accuracy }\end{array}$ & $\begin{array}{l}\text { To compensate for increase } \\
\text { latency in command and control } \\
\text { of UAS in BLOS operations, } \\
\text { assured navigation accuracy is } \\
\text { required to allow for increased } \\
\text { autonomous functions. }\end{array}$ \\
\hline $\begin{array}{l}\text { NV7: Autonomous } \\
\text { Accuracy }\end{array}$ & $\begin{array}{l}\text { Autonomous UAS landing on a } \\
\text { stationary landing pads or } \\
\text { runways will require UAS sensor } \\
\text { capabilities to augment the } \\
\text { human similar to manned aircraft } \\
\text { during required visual phases of } \\
\text { landing. }\end{array}$ \\
\hline
\end{tabular}

\section{Onboard UAS Architectural Framework}

The onboard UAS architecture concept is approached with the consideration that "no one stand-alone technology" is envisioned to augment GPS for all customers and all operational conditions. Therefore, the Boeing team is recommending an architectural framework which can evolve and support a wide range of alternative navigation sources to address the requirement "NV1: GPS Augmentation". Figure 8 shows a recommended navigation architecture framework to exploit navigation technologies and techniques for augmenting GPS: with line replaceable units (LRU) to software partitions within a consolidated hardware package, such as System on Chip (SoC).

This architectural consideration is supportive of modular open architecture for ease of integration of a vast number of sensors either dedicated to navigation or leveraged from surveillance and/or communications sources. ARINC 653 can support mix criticality of applications from nonessential to flight safety critical within the same computing hardware which addresses the requirement NV2: Certifiable Navigation Computing Architecture."

\section{Integration Perspectives}

GPS signals alone are extremely weak due to high frequency and useless in certain environments, such as low urban and deep canyon operations. To address requirement "NV3: Navigation Source Error Detection and Correction" during GPS-denied or degraded conditions, the use of both recommended architectural framework and the IMA computing based on ARINC 653 can provide a means of real-time navigation error detection and correction. Within ARINC 653 there are a set of Health Monitoring features which can be utilized to detect an error with either the GPS hardware or quality of the measurement. The correction would then be handled by the ARINC 653 configuration table which would be defined dependent on the type of errors expected and types of navigation source alternatives available.

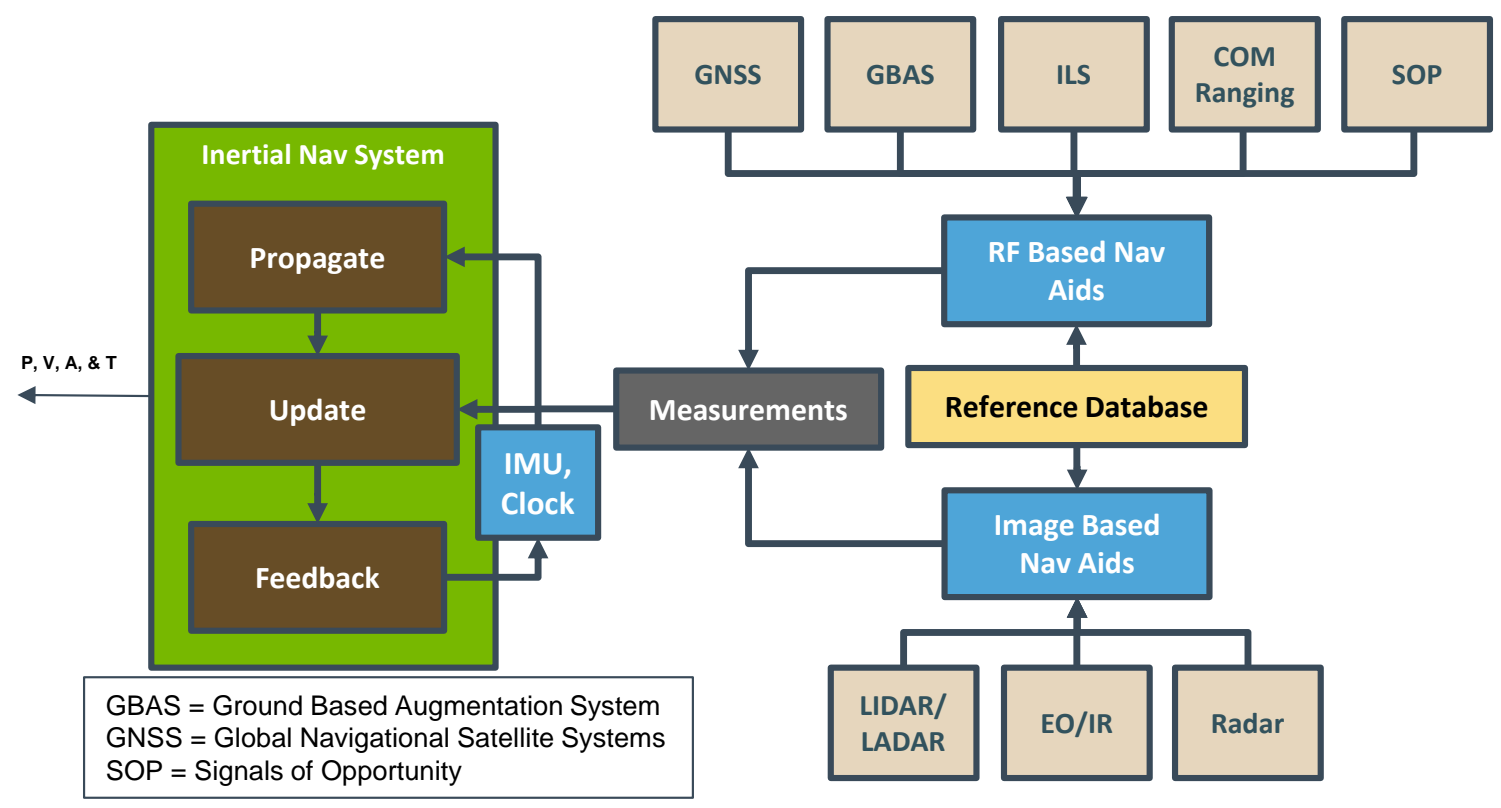

Figure 8- UAS Navigation Architectural Framework

\section{IMA Partitioning}

Boeing recommends utilizing Integrated Modular Avionics (IMA) techniques supporting onboard navigation integration to reduce cost, weight, space, and power consumption. This architectural concept would be useful for all airborne platforms to move away from the traditional integration
The recommended architectural framework and the IMA computing based on ARINC 653 is supportive of all flight phases of an UAS. For example, navigation algorithms can be developed within separate partitions supporting "NV4: Ground Controlled UAS Navigation Accuracy" and "NV6: BLOS Navigation Accuracy" requirements for different flight phases accommodating the vast number of UAS 
maneuverability (e.g., speed, rate of turns, climb, descent, etc.) profiles and the varying closed loop command and control time for Line of Sight (LOS) and Beyond Line of Sight (BLOS) communications.

For autonomous landing, "NV7: Autonomous Landing Navigation Accuracy" requirement, the recommended architectural framework and the IMA computing based on ARINC 653 are supportive of tightly coupled navigation algorithms with flight control algorithms. This technical approach would be very similar to fielded auto-pilot systems on manned platforms.

As a means to integrate within ATM, the UAS onboard architectural framework is envisioned to communicate using an industry approach message schema supporting "NV5: Universal Navigation Message Schema." The recommended schema is based on STANAG 4586 to exchange navigation information between the UAS and respective ground station.

\section{UAS Navigation Architecture Summary}

In summary, the onboard UAS navigation architecture concept is approached leveraging multiple sources with a minimalistic addition of equipage with the consideration that "no one stand-alone technology" will augment GPS in all flight phases in Class A - E airspace. The proposed architecture is envisioned to host functions beyond navigation, such as surveillance, communications, vehicle management, flight controls, maintenance, etc., with the use of the IMA computing architecture based on ARINC 653. The UAS navigation architecture concept is also envisioned supporting navigation functions by leveraging sensors for non-cooperative detect and avoid capabilities and signal characteristics from onboard communications systems.

\section{SURVEILLANCE}

\section{Introduction}

Controlled airspace is defined by a set of requirements in terms of altitude, proximity to airports, ATC clearances, avionics, instrumental flight rules, and visual flight rules [11]. An efficient UAS ATM system requires cooperative surveillance systems. In particular, dependent cooperative surveillance systems provide significant benefits including high precision independent of target distance, transmission of additional data such as velocity [12]. UAs must implement methods to autonomously determine their own positions.

This section of the presents ADS-IP system, a proposal of cooperative surveillance system able to cope with the upcoming paradigm of UAS air traffic and to overcome the limitations of current surveillance systems for controlled airspace.

\section{The need of UAs of being connected}

Although it is possible to carry on completely autonomous UAs missions, from a safety perspective, it is imperative to implement such communication means. There shall always be a pilot responsible for the flight who, in case of emergency or under any other circumstances, can remotely take control of the vehicle. An RF-based communication channel is usually used for this purpose.

Apart from that, it is considered necessary a more accessible media to enable the monitoring of the UA and to enable potential interventions over it. The kind of data links required for these kind of services can be established through Internet connections.

\section{Why ADS-IP}

Traditional surveillance systems are already close to saturation. Current surveillance systems based on RF transmissions will not be able to cope with the upcoming UAS paradigm.

Alternative surveillance systems, such as ADS-IP (Figure 9), shall be developed to overcome most of the drawbacks of current surveillance systems (saturation, propagation, security).

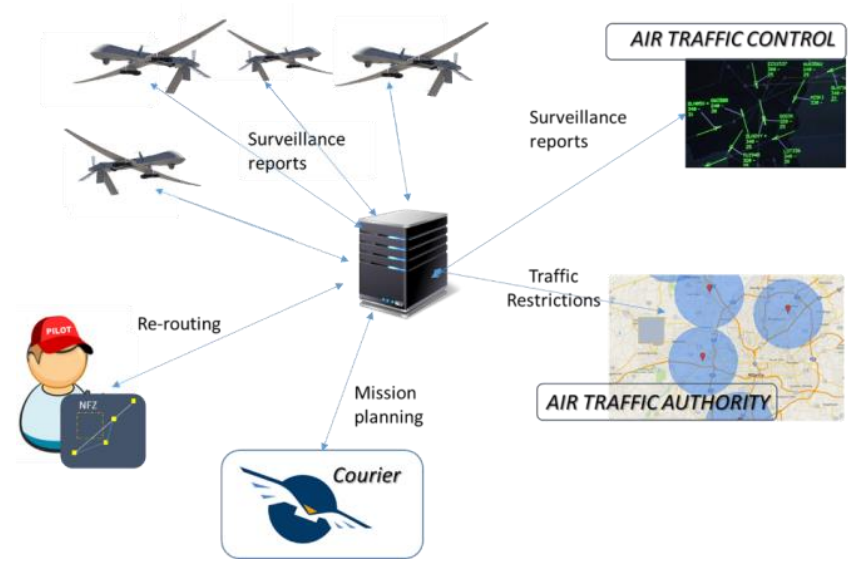

Figure 9 - ADS-IP Surveillance System

The use of a system like ADS-IP expands the capabilities and features of traditional surveillance systems. ADS-IP provides a series of additional services.

\section{ADS-IP functional description}

ADS-IP is a centralized, automated, and cooperative surveillance system. ADS-IP uses IP transmission channels to manage the data interchange between UAs and a server on the ground, and between such server and other actors such as an automatic traffic supervisor or the fleet owner. A server on ground acts as the core of the system, gathering all the navigation data transmitted by the UAs and distributing it accordingly to the needs of each actor. 
The range of the system is established by artificial means. For each UA an area of interest is established. Areas of interest are customizable in shape and size. Each UA receives surveillance data from the air traffic within its area of interest.

Each ADS-IP equipped UAS, through IP channels, sends its surveillance data to a server on ground asynchronously and with a determined rate. Surveillance data include the aircraft ID, its location and other parameters such as air speed or intent.

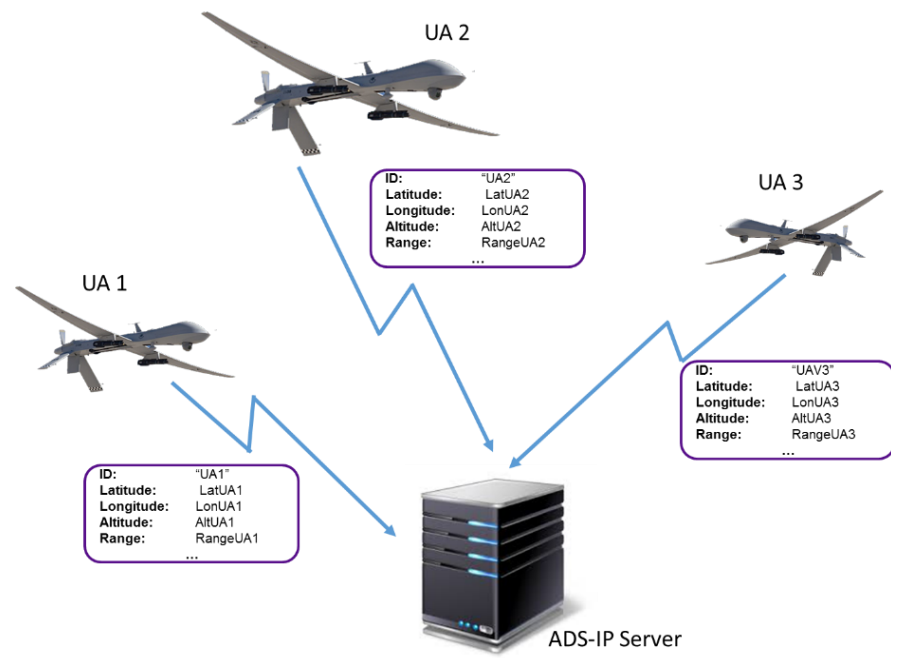

Figure 10 - ADS-IP Server Gathering Surveillance Information from UAs

The ADS-IP server is in charge of gathering and storing the information received from all the UAs (Figure 10). The server analyzes, processes the information received, and determines what information shall be relayed to each UA.

The ADS-IP server is in charge of distributing the surveillance data not only to the flying UAs (Figure 11) but also to other entities that also need the information for their operations (e.g., ATC, fleet owner).

\section{Geo-segmentation}

Each ADS-IP server has its own area of service (Figure 12). ADS-IP servers (AS) receive surveillance data from UAs flying within their area of service. These areas are customizable for each AS, in order to cover its area of service. To provide decentralization, multiple AS need to be deployed.

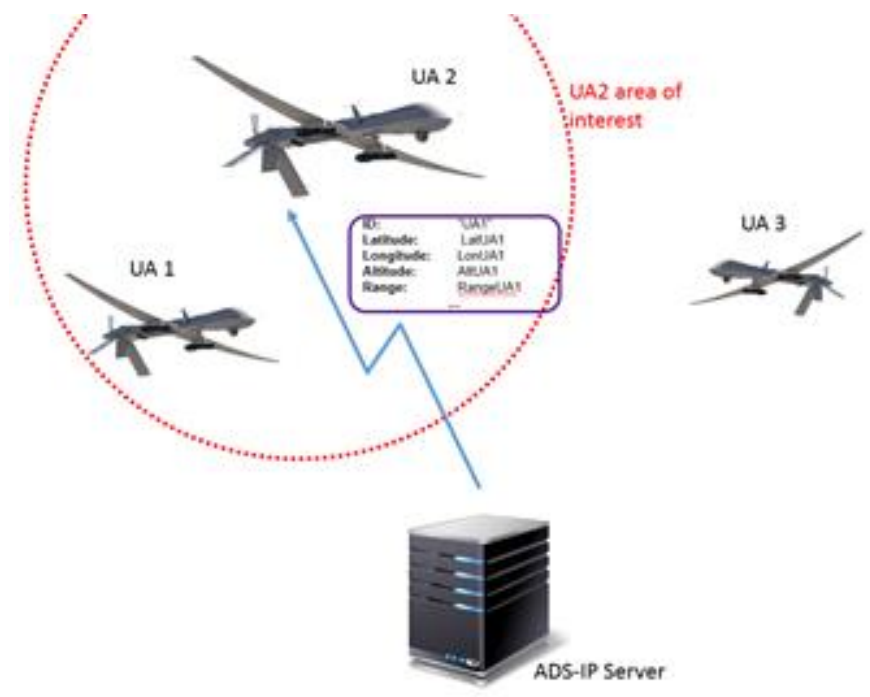

\section{Figure 11 - ADS-IP Server Distributing Surveillance Information to UAs}

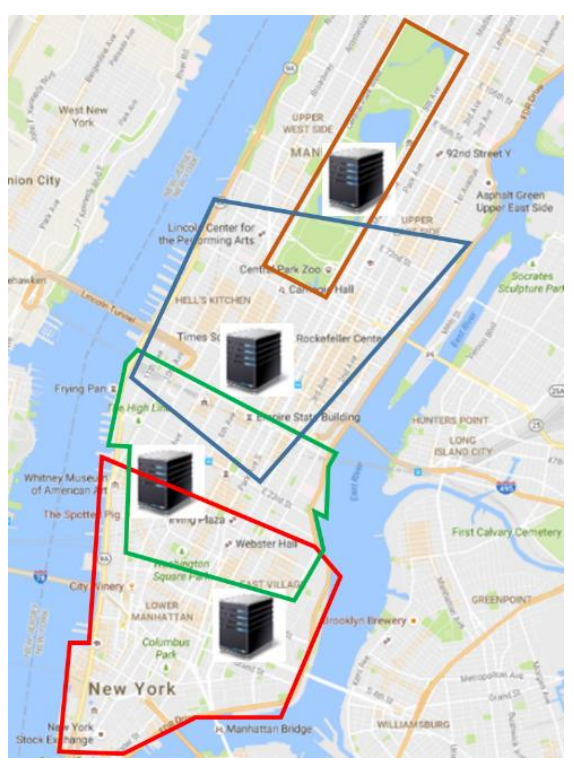

Figure 12- Areas of Interest

With decentralization, some issues arise. UAs need to know to which AS shall establish the connection in order to start sending and receiving surveillance data. To solve it, ADS-IP architecture deploys a series of ADS-IP Directory Servers (DS) hierarchically and geographically organized. They provide the information of what is the AS to be used depending on the position and other parameters (e.g., heading, speed, etc.). Each DS has a database of ADS-IP Servers and their service areas (Figure 13). DS receive queries from UAs and they return a list of AS sorted following a criterion to determine which is the most convenient AS for each UAS for a determined situation. 


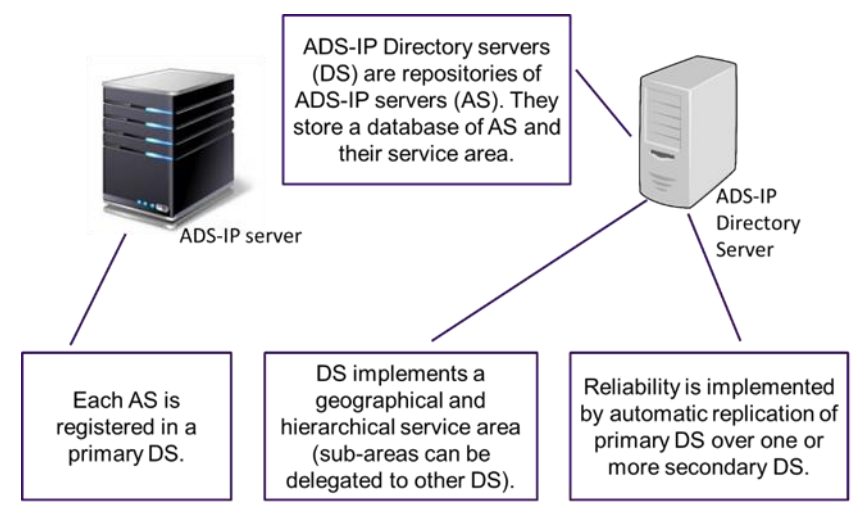

Figure 13 - ADS-IP Directory Service

ADS-IP servers are registered into a specific DS (primary). Each primary DS replicates the information within its database over one (or more) secondary DS in order to enhance the reliability of the ADS-IP system.

UAs are able to query any DS. Queries over DS are forwarded to the correspondent DS if the location of the UA belongs to a different DS service area.

The descriptive process of the overall performance of ADSIP is as follows:

1. An UA autonomously determines its location.

2. It queries to a DS for a list of ADS-IP servers within its area.

3. The DS determines a list of usable ADS-IP servers and provides the UA with it.

4. The UA then establishes a connection with one of the ADS-IP servers of the list.

Other issues related with the geo-segmentation of ADS-IP are:

- Handovers: To deal with handovers, AS sends an alert to the UAS when it is close to the boundary of its area of service. The UAS queries a DS again.

- Overlapping areas: It is necessary to establish coordination mechanisms between overlapping AS.

\section{Services that can be delivered by ADS-IP}

This section presents a series of services that can be provided by ADS-IP:

- Surveillance data gathering and broadcast: This is the main function of the ADS-IP system.

- Tracking services: ADS-IP servers deploy data persistence capabilities which enables ADS-IP to provide non-real time data tracking services and analytics to operators, authorities...
- Dynamic exclusive/inclusive fly zones: Through ADS-IP it is possible to create exclusive and inclusive fly zones.

- Cooperative anti-collision systems: ADS-IP analyzes the tracking of the UAs in real time and predicts the future location of UAs, so by this mean it is also possible to predict safety issues.

\section{ADS-IP Security}

This section presents how ADS-IP overcomes the main vulnerabilities of ADS-B [13] and as shown in Table 2.

Table 2 - Threats Addressed by ADS-IP

\begin{tabular}{|c|c|}
\hline Threats & ADS-IP Security \\
\hline Eavesdropping & Message Encryption \\
\hline Jamming & Multilink \\
\hline Message injection & Authentication mechanisms \\
\hline Message deletion & Acknowledge Mechanisms \\
\hline Message modification & Signatures \\
\hline
\end{tabular}

ADS-IP relies on secure transmission channels. The communications infrastructure establishes a series of encrypted VPNs. Thus, eavesdropping will not be feasible.

ADS-IP does not use the $1090 \mathrm{MHz}$ frequency band. As the integral architecture proposed relies on the establishment of communication channels through different data links, the risk of a denial of service based on jamming is decreased.

ADS-IP implements authentication mechanisms to avoid fraudulent injection of messages.

ADS-IP uses acknowledge mechanisms and encryption techniques to sign the messages; these measures reduce the risk of successful message injection and message deletion attacks.

\section{Modes of operation of ADS-IP}

ADS-IP implements two different modes of operation, authenticated and non-authenticated.

An authenticated mode is implemented in order to verify the identity of the UAs. To operate in this mode, each UA shall be registered following the procedures established by regulations. In order to facilitate the registration procedure, an auto-provisioning system may be implemented. This mode of operation reinforces the confidentiality, integrity, authenticity, and non-repudiation dimensions of the ADS-IP system. On the other hand, an authenticated system presents some issues. The process of registration might be seen as an entry barrier by the users of the system. It will be necessary to define new roles and responsibilities to maintain the authentication system. Therefore, there shall be developed and implemented systems to solve the problem of authenticating UAs over different ADS-IP systems of different service areas or jurisdictions. 
An open mode of operation is also possible. In this mode of operation, both ADS-IP servers and Directory servers are publicly available and provide surveillance services without requesting any credentials to the UAs. At first glance, this mode of operation can be seen as an insecure system. The system might be vulnerable to spoofing and DoS attacks. However, there are several countermeasures that can be applied to minimize these risks. On the other hand, a surveillance system working in an open mode presents some interesting benefits. The main one is that a higher number of UAs might be monitored, raising the safety level of the air traffic. With this mode of operation, the problems related to the management of the credentials disappear, reducing the costs of maintenance and increasing the compatibility between ADS-IP servers of different owners.

\section{ADS-IP pros and drawbacks}

ADS-IP Pros - This section of the document highlights a series of features of ADS-IP which improve the current capabilities of current existing surveillance systems in controlled airspaces.

- Global Tracking. One of the main problems of current surveillance systems is the lack of coverage outside the operational areas of radar, ADS-B or multilateration.

- Security. ADS-B vulnerabilities are well known as they have been proved and presented in various papers available on the public media [14].

- Integration of UAS in the airspace. The UAS industry is growing and it is expected that UAs will be sharing airspace with commercial air traffic. Surveillance technologies are needed for a safe integration of UAs in the airspace without reducing existing capacity.

ADS-IP Drawbacks - This section presents the identified limitations of ADS-IP.

- Integration with current surveillance systems: When deploying ADS-IP within controlled airspace, it will be necessary to invest an important amount of effort in the integration of ADS-IP with current surveillance systems used by the ATCs to manage the air traffic.

\section{Cooperative surveillance systems limitations}

ADS-IP share some limitations with the rest of cooperative surveillance systems. There is still a need to develop surveillance systems to detect, identify, and cope with noncompliant systems (non-intentional and intentional).

\section{SUMMARY}

In this document, we propose revolutionary new architectural concepts for Communications, Navigation and
Surveillance (CNS) of UAS in controlled airspace. The ideas have implications for standards organization activities such as in the IEEE, IETF, ICAO and RTCA, and further build on designs that have been under development in internal R\&D efforts in the authors' organizations. We believe that these concepts can help open new opportunities for the safe operation of UAS in controlled airspace in cooperation with the Air Traffic Management systems both within the United States and world-wide.

We see the emergence of a worldwide ATN/IPS service with networks and data links that can support data communications for UAS ATM as a key enabler to allow safe integration of UAS in non-segregated airspace. We further believe the new concepts in navigation and surveillance presented here will be instrumental in maintaining safe operations in cooperation with manned aviation.

The next phase of our investigation will explore new UAS CNS architectural concepts for the operation of small UAS (sUAS) in uncontrolled airspace. These concepts will be in keeping with the UTM Concept of Operations articulated by NASA [2] and that is being embraced by the worldwide UAS community. One of the first questions to be answered is whether the UTM system will be separate from the system proposed here for controlled airspace, or will it all be a single system for both.

Clearly, ATC/AOC workload must be a primary consideration given that there will be millions of UAS operating in uncontrolled airspace in the coming years. The key, therefore, is to again allow the requirements to shape the architectural solutions we will propose. We believe that the same concepts developed here for UAS operation in controlled airspace can be adapted for operation of sUAS in uncontrolled airspace with the key differences in scale (i.e., the numbers of sUAS in operation) as well as vehicle size weight and power.

\section{ACKNOWLEDGEMENTS}

This work is aligned with the NASA Safe Autonomous Systems Operation (SASO) program under NASA contract number NNA16BD84C.

\section{REFERENCES}

[1] "ICAO 9896 Manual on the Aeronautical Telecommunication Network (ATN) Using Internet Protocol Suite (IPS) Standards and Protocols", Second Edition 2015.

[2] Kopardekar, P., et al., "Unmanned Aircraft System Traffic Management (UTM) Concept of Operations", AIAA Aviation Technology, Integration, and Operations Conference, June 2016. 
[3] Templin, F. "Asymmetric Extended Route Optimization (AERO)”, draft-templin-aerolink, (work-in-progress), May 2017.

[4] Raj Jain, Fred L. Templin, "Datalink for Unmanned Aircraft Systems: Requirements, Challenges and Design Ideas," AIAA Infotec@Aerospace Conference, Saint Louis, MO, March 2011, http://www.cse.wustl.edu/ jain/papers/uas_dl.htm

[5] Raj Jain, Fred L. Templin, Kwong-Sang Yin, "Analysis of L-Band Digital Aeronautical Communication Systems: LDACS1 and L-DACS2," 2011 IEEE Aerospace Conference, Big Sky, Montana, March 5-12, 2011, pp. 110, http://www.cse.wustl.edu/ jain/papers/ldacs.htm

[6] Raj Jain and F. Templin, "Requirements, Challenges and Analysis of Alternatives for Wireless Datalinks for Unmanned Aircraft Systems," IEEE Journal on Selected Areas in Communications (JSAC) Special Issue on Communications Challenges and Dynamics for Unmanned Autonomous Vehicles, Vol. 30, No. 5, June 2012, pp. 852-860, http://www.cse.wustl.edu/ jain/papers/uas_jsac.htm

[7] RTCA, Operational Services and Environmental Definition (OSED) for Unmanned Aircraft Systems (UAS), DO-320, 2010, 236 pp.

[8] RTCA, UAS Command and Control (C2) Data Link White Paper, WP-2_C2, 2014, 88 pp.

[9] P. Zhu, "5G Enabling Technologies," PIMRC, Sep 2014, 20 slides, http://www.ieee-pimrc.org/2014/2014-0903\%205G\%20Enabling\%20Technologies\%20PMIRC\%2 OHuawei Final.pdf

[10] Impact and Mitigation of GPS-Unavailability on Small UAV Navigation, Guidance and Control, https://core.ac.uk/download/pdf/11432788.pdf

[11] FAA NY/NJ/PHL Airspace Redesign Final Environmental Impact Statement (FEIS), "Appendix A: National Airspace System Overview.” July 2007. 5pp.

[12] RTCA, "Minimum Aviation system Performance Standards for Automatic Dependent SurveillanceBroadcast (ADS-B)," DO-242A, June 25, 2002, 475 pp.

[13] Martin Strohmeier, et al., "On the Security of the Automatic Dependent Surveillance-Broadcast Protocol," April 15, 2014.

[14] Andrei Costin, Aurelien Francillon. "Ghost in the Air(Traffic): On insecurity of ADS-B protocol and practical attacks on ADS-B devices." Network and Security Department EURECOM Sophia-Antipolis, France. Black Hat USA, 2012. 16, 2012.

\section{Biographies}

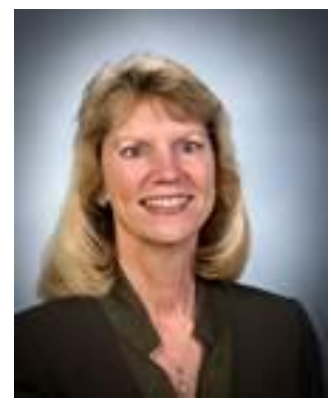

Denise S. Ponchak is Branch Chief of the Communications Architectures, Networks and Systems Branch at the National Aeronautics and Space Administration's (NASA) Glenn Research Center at Lewis Field in Cleveland, Ohio. The Branch is responsible for designing advanced networking concepts, architectures, and technologies for aeronautics and space applications. Prior to becoming Branch Chief, Ms. Ponchak was an Aeronautical Communications Project Manager focusing on increasing the National Airspace System's telecommunications capability, and a Communications Research Engineer supporting future satellite-based communications. She holds a Bachelor's of Electrical Engineering and a Master's of Science in Electrical Engineering from Cleveland State University in 1983 and 1988 respectively.

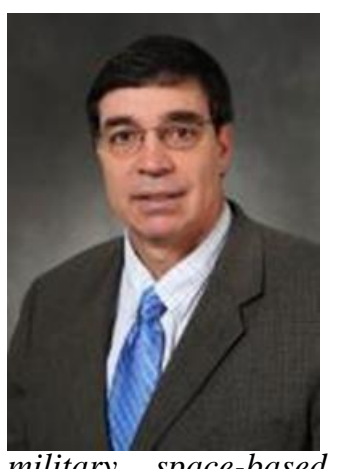

Fred Templin is a computer networking $R \& D$ professional with a focus on Internet protocol and data link specifications, operating system networking internals, networked applications, and networked platforms. He has indepth experience in Internet networking and security architectures for unmanned air systems, civil aviation, tactical military, space-based systems and enterprise network applications. Mr. Templin has been an active contributor to the Internet Engineering Task Force (IETF) since 1999. He is currently a senior research engineer in Boeing Research \& Technology (BR\&T) since May 2005, where he is an Associate Technical Fellow of The Boeing Company.

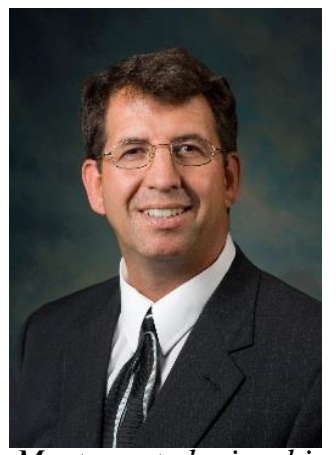

Greg L. Sheffield is a Senior Research Engineer in the Boeing Research \& technology (BR\&T) Avionics Systems Technology group. Greg's experience includes over 26 years working with commercial and defense communications, navigation, and surveillance technologies and products. He has contributed to a number of IEEE, RTCA, ARINC, and SAE standards. Most noted is his contributions related to digital communications using ACARS, second-generation TCAS combined with ADS-B, Navigation solutions in denied or degraded environments, and affordable open avionics architectures solutions. Greg is a retired Navy Flight Officer and holds BSEE, BSCS, SysEngMS, and MBA degrees. He lives and works in the St Louis, MO area. 


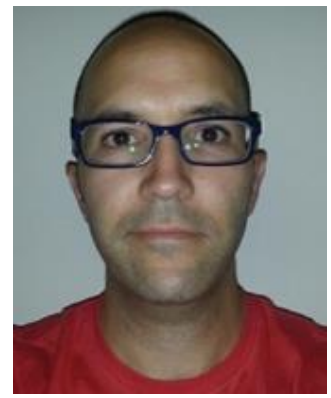

Pedro Taboso is a Telecommunication Engineer (5 year-degree, Polytechnic University of Madrid) with a background in IT systems and a proven professional and hands-on experience within Cyber Defence and Information Assurance fields. Pedro holds relevant security trainings and certifications such as CISA (Certified Information Security Auditor), ITILv3 and SANS 508 (Computer Forensics). He has a strong background on Information Security projects (penetration testing, ethical hacking, security architecture design, risk analysis...). During the last four years, he has been working on applications development for "Security- Over- CNS systems" projects, defining and developing new Surveillance systems for both manned and unmanned aircraft.

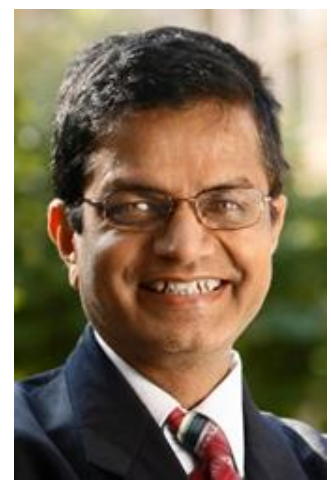

Raj Jain is a Fellow of IEEE, ACM, and AAAS. He is a winner of 2017 ACM SIGCOMM Life-Time Achievement Award, 2015 A.A. Michelson Award. Dr. Jain is currently the Barbara J. and Jerome R. Cox, Jr., Professor of Computer Science and Engineering at Washington University in St. Louis. Previously, he was one of the Co-founders of Nayna Networks, Inc - a next-generation telecommunications systems company in San Jose, CA. He was a Senior Consulting Engineer at Digital Equipment Corporation in Littleton, Mass and then a professor of Computer and Information Sciences at Ohio State University in Columbus, Ohio. 